\title{
Special Calixarenes Incorporating Sulfonamide Moieties: Versatile Ligands for Carbonic Anhydrases Inhibition
}

\author{
Davide Sbravati, ${ }^{[\mathrm{a}]}$ Alessandro Bonardi ${ }^{[\mathrm{b}, \mathrm{c}]}$ Silvia Bua, ${ }^{[\mathrm{b}]}$ Andrea Angeli, ${ }^{[\mathrm{b}]}$ Marta Ferraroni, ${ }^{[\mathrm{d}]}$ \\ Alessio Nocentini, ${ }^{[b, c]}$ Alessandro Casnati, ${ }^{[a]}$ Paola Gratteri, ${ }^{*[b, c]}$ Francesco Sansone, ${ }^{*[a]}$ and \\ Claudiu T. Supuran ${ }^{[b]}$
}

Dedicated to Vincenzo Balzani on the occasion of his 85th birthday

\begin{abstract}
Carbonic anhydrases (CAs) continue to represent a relevant pharmaceutical target. The need of selective inhibitors and the involvement of these metalloenzymes in many multifaceted diseases boost the search for new ligands able to distinguish among the different $C A$ isoforms, and for multifunctional systems simultaneously able to inhibit CAs and to interfere with other pathological events by interacting with additional targets. In this work, we successfully explored the possibility of preparing new CAs ligands by combining
\end{abstract}

\section{Introduction}

Carbonic anhydrases (CAs) ${ }^{[1]}$ are metalloenzymes ubiquitously expressed in tissues, where play a crucial role in many processes and pathways such as $\mathrm{pH}$ regulation, lipogenesis, gluconeo-

[a] Dr. D. Sbravati, Prof. Dr. A. Casnati, Prof. Dr. F. Sansone

Department of Chemistry,

Life Sciences and Environmental Sustainability

University of Parma

Parco Area delle Scienze 17/A,

43124 Parma (Italy)

E-mail: francesco.sansone@unipr.it

[b] Dr. A. Bonardi, Dr. S. Bua, Dr. A. Angeli, Dr. A. Nocentini, Prof. Dr. P. Gratteri, Prof. Dr. C. T. Supuran

Department of NEUROFARBA

Section of Pharmaceutical and Nutraceutical Sciences University of Florence

Polo Scientifico, Via U. Schiff 6, 50019

Sesto Fiorentino, Firenze (Italy)

E-mail: paola.gratteri@unifi.it

[c] Dr. A. Bonardi, Dr. A. Nocentini, Prof. Dr. P. Gratteri Department of NEUROFARBA

Section of Pharmaceutical and Nutraceutical Sciences

Laboratory of Molecular Modeling Cheminformatics \& QSAR

University of Florence

Polo Scientifico, Via U. Schiff 6, 50019

Sesto Fiorentino, Firenze (Italy)

[d] Dr. M. Ferraroni

Department of Chemistry "Ugo Schiff"

University of Florence

Via della Lastruccia 3, 50019 Sesto Fiorentino, Florence (Italy)

$\square$ Supporting information for this article is available on the WWW under https://doi.org/10.1002/chem.202103527

Special
colection This manuscript is part of a special collection dedicated to Vincenzo Balzani on the occasion of his 85th birthday. calixarenes with benzensulfonamide units. Inhibition tests towards three human CA isoforms evidenced, for some of the ligands, $K_{i}$ values in the nanomolar range and promising selectivity. X-ray and molecular modeling studies provided information on the mode of binding of these calixarene derivatives. Thanks to the encouraging results and the structural features typical of the calixarene scaffold, it is then possible to plan for the future the design of multifunctional inhibitors for this class of widely spread enzymes.

genesis and ureagenesis. ${ }^{[2]}$ Their alteration and overexpression is related to many diseases of variable severity and incidence. ${ }^{[3]}$ Effective inhibitors of their enzymatic activity show anticancer, antiglaucoma, diuretics, antiobesity properties, or result useful in the treatment and management of several neurological disorders, including epilepsy. ${ }^{[4]}$

This wide involvement in numerous diseases suggests the possibility of inhibiting them with hybrid and multifunctional molecular systems able to simultaneously and synergistically perform further activities against other altered processes and biological entities concause of the pathology. For example, a series of molecules were proposed with activity against rheumatoid arthritis ${ }^{[5]}$ or inflammation in human tenocytes, ${ }^{[6]}$ consisting of inhibitor moieties for CAs covalently linked to carbon monoxide releasing complexes. Neri et al. developed compounds that elicit complete tumor regression in mice, by connecting to a common core the sulfonamide unit for CA IX targeting and the potent cytotoxic maytansinoid DM1. ${ }^{[7]}$ Analogously, treatment of tumors could be more beneficial if photosensitizers for photodynamic therapy were combined with inhibitors of CA IX because of its relationship with angiogenesis and its promoting ability of cancer cell survival and invasion within hypoxic environments. ${ }^{[8]}$ Inhibitory units for CA II and/or CA VII could be linked together with inhibitory units for $\mathrm{N}$-acetylgalactosamine-6 sulfatase $(\mathrm{GALNS})^{[9]}$ to a common core, being both enzymes involved in neuropathic pain. Also from a diagnostic point of view, combination of different functions including CAs binding species would be interesting as shown with a ${ }^{129} \mathrm{Xe}$-Cryptophane Biosensor for Nuclear Magnetic Resonance. ${ }^{[10]}$

Another important issue is that, despite the existence of approved drugs acting as CAs inhibitors and the 40-year 
worldwide research in this field, selectivity is still a challenge and an important goal to reach. ${ }^{[4 a, 11]}$ The selective inhibition of single CAs isoforms among the fifteen human ones or the selectivity between human and microbial CAs are strongly desired. Rather recently, the possibility that Multivalency ${ }^{[12]}$ can play an important role also in enzyme binding/inhibition has been proposed and verified. ${ }^{[13]}$ This phenomenon could, indeed, not only improve the inhibition efficiency but also help in determining selectivity among the different CAs isoforms. Few recent examples of multivalent systems designed for these purposes have been reported in the last years. ${ }^{[14]}$ For instance, gold nanoparticles exposing multiple benzensulfonamide units showed selectivity for cancer related CA IX over other CAs. ${ }^{[15]}$ The above mentioned compound by Neri et al. ${ }^{[7]}$ is actually divalent and also fullerenes displaying multiple copies of coumarin as inhibiting units were proposed and studied as CAs ligands. ${ }^{[16]}$

In line with these approaches, we herein report our explorative work on the use of calixarenes as scaffolds for the design of CAs inhibitors. Calixarenes demonstrated, in fact, to be excellent platform for the building of ligands showing strong positive effects related to both the multiple exposition of epitope units and the macrocyclic preorganized nature in the binding of proteins, ${ }^{[17]}$ in particular carbohydrate binding proteins (lectins), ${ }^{[18]}$ and nucleic acids. ${ }^{[19]}$ Moreover, in perspective, they appear suitable to design multifunctional systems $s^{[20]}$ where further active functions can be assembled in addition to the CA inhibitory moieties, thanks to the non-covalent inclusion into the macrocyclic cavity and/or a rather easy covalent functionalization at the two rims, even in a multivalent arrangement.

A paper ${ }^{[21]}$ reporting calix[4]arene-azacrown-based ligands for CAs inhibition was recently published. Five compounds out of six presented two secondary sulfonamide units and one two primary sulfonamide groups. However, only this latter showed a rather significant activity and no rational for the binding to the enzymes was reported.

Selecting a primary benzenesulfonamide as the inhibitory unit $^{[22]}$ we have prepared a series of twelve novel calixarene derivatives, differing for the number of sulphonamide units attached to the macrocycle $(1,2,4$ or 6$)$, the size of the calix[n]arene $(n=4,6)$ and its conformation. They exhibited promising and, in some case, selective inhibitory activity towards some hCAs. To ascertain the occurrence of a multivalent effect, we have also synthesized acyclic mono- and divalent (gemini) derivatives based on simple phenol units.

\section{Results and Discussion}

Synthesis of the inhibitors. We performed the synthesis of calix[4]- and calix[6]arene derivatives (Scheme 1) bearing a selected number of benzenesulfonamide units at the lower rim tethered to the macrocyclic scaffold through a propyl chain and connected via thiourea or amide moieties. To this aim, we started from the $\mathrm{N}$-phthalimidopropyl derivatives $\mathbf{1}$ a-f that were deprotected to amines $\mathbf{2} \mathbf{a}-\mathbf{f}$, in turn reacted with the

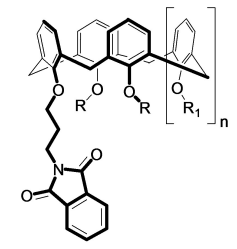

1a: $\mathrm{n}=1, \mathrm{R}=\mathrm{R}_{1}=$ propyl 1b: $n=1, R=R_{1}=$ ethoxyethy 1c: $n=1, R=$ propyl, $\mathrm{R}_{1}=\mathrm{N}$-propylPh 1d: $n=1, R=$ ethoxyethyl, $R_{1}=N$-propylP if: $n=3, R=R_{1}=N$-propylPht

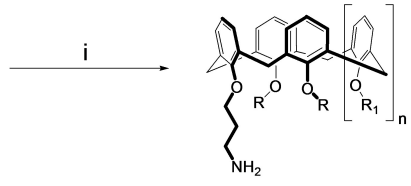

2a: $\mathrm{n}=1, \mathrm{R}=\mathrm{R}_{1}=$ propy 2b: $\mathbf{n}=1, \mathrm{R}=\mathrm{R}_{1}=$ ethoxyethyl 2c: $n=1, R=$ propyl, 2d: $n=1, R=$ ethoxyethyl,

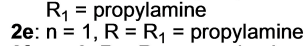
2f: $n=3, R=R_{1}=$ propylamine

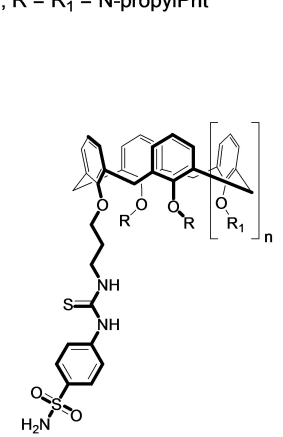

4b: $\mathrm{n}=1, \mathrm{R}=\mathrm{R}_{1}=$ ethoxyethyl 4c: $n=1, R=$ propyl,

$\mathrm{R}_{1}=$ propylthioureidosulfonamide 4d: $n=1, R=$ ethoxyethyl,

$\mathrm{R}_{1}=$ propylthioureidosulfonamide 4f: $n=3, R=R_{1}=$ propylthioureidosulfonamide
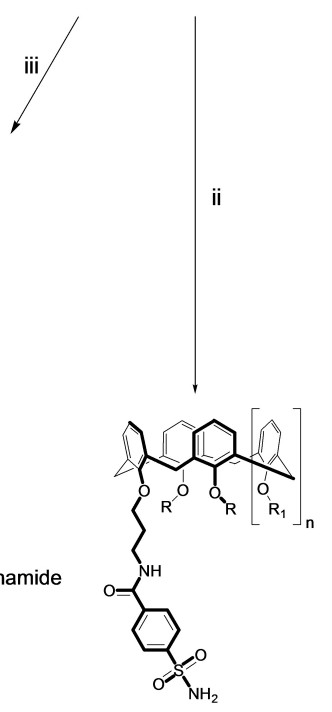

3a: $n=1, R=R_{1}=$ propyl

$3 \mathbf{b}: \mathbf{n}=1, \mathrm{R}=\mathrm{R}_{1}=$ ethoxyethy 3c: $n=1, R=$ propyl,

$\mathrm{R}_{1}=$ propylamidosulfonamide

3e: $n=1, R=R_{1}=$ propylamidosulfonamide 3f: $n=3, R=R_{1}=$ propylamidosulfonamide

Scheme 1. Reagents and conditions: i) $\mathrm{NH}_{2} \mathrm{NH}_{2} \cdot \mathrm{H}_{2} \mathrm{O}$, abs EtOH, reflux; ii) 4sulfamoylbenzoic acid, DIPEA, EDC; iii) 4-isothiocyanatebenzensulfonamide, DIPEA.

proper sulfonamide reagents to obtain the amide ligands $\mathbf{3 a}-\mathbf{c}, \mathbf{e}, \mathbf{f}$ and the thioureido derivatives $\mathbf{4} \mathbf{b}-\mathbf{d}, \mathbf{f}$ (see Figure S1 for explicit structure of these sulfonamide containing calixarenes). While calix[4]arenes are blocked in the cone geometry, because of the experimental conditions exploited for the lower rim functionalization, calix[6]arenes are conformationally mobile.

To investigate the impact on the inhibition properties of a different stereochemical arrangement of the benzensulfonamide units in the space, in particular with respect to the tetravalent ligand $3 \mathrm{e}$ in cone geometry, the 1,3-alternate calix[4]arene 7 was also prepared (Scheme 2). From calix[4]arene the tetraazide $\mathbf{5}$ was prepared and transformed into the corresponding tetraamino derivative 6 . This was reacted with 4-isothiocyanatebenzensulfonamide to give 7 . Actually, $\mathbf{7}$ and $\mathbf{3 e}$ differ also for the connecting unit between sulfonamide moiety and propyl chain, being a thiourea in the former and an amide in the latter. Unexpectedly, in fact, attempts to obtain the analogue of 7 containing the amide connection failed. 


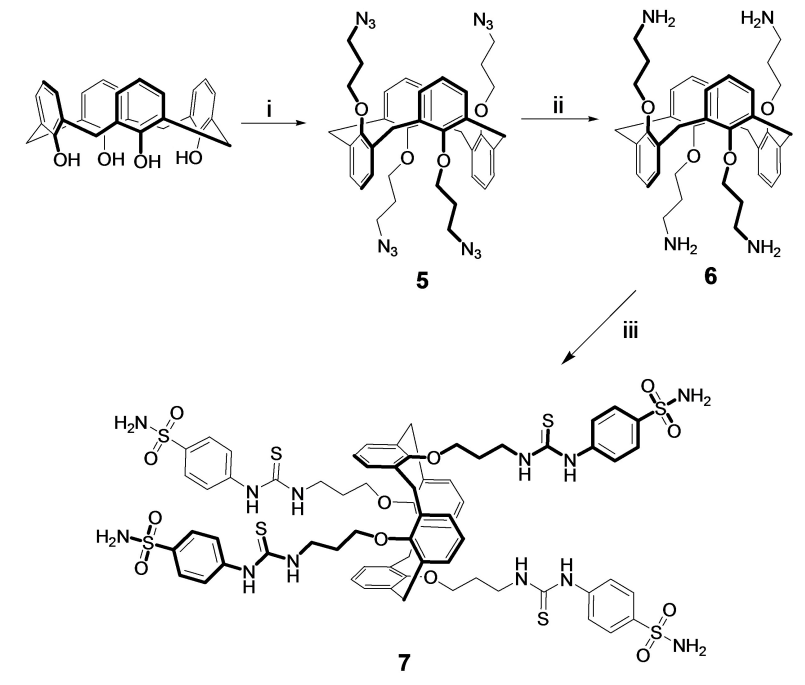

Scheme 2. Reagents and conditions i) $\mathrm{Cs}_{2} \mathrm{CO}_{3}$, 1-iodo-3-azidopropane, dry DMF, rt, 3 days; ii) $\mathrm{PPh}_{3}$, dry $\mathrm{CHCl}_{3}$, rt, 24 h; iii) 4-isothiocyanatebenzensulfonamide, DIPEA, dry DMF, rt, 1 day.

To verify a possible multivalent effect and/or a role of the macrocyclic skeleton in the inhibition of the enzymes, we synthesized two monovalent derivatives (9 and 10, Figure 1) based on a simple phenol unit. They represent the monomeric moiety constituting the macrocyclic derivatives used as reference compounds in the inhibitory tests. The two monomeric compounds were obtained by conjugating the benzenesulfonamide unit to 1-phenoxy-3-aminopropane (8). Namely, derivative 9 was synthesized by reaction with 4-isothiocyanate benzensulfonamide generating a thiourea connecting spacer, while derivative 10 was prepared through the formation of an amide bond by reaction with 4-sulfamoylbenzoic acid in the presence of EDC as coupling reagent.

With the same purposes, we also synthesized the thioureido, divalent gemini derivative 11 (Figure 1) representing the half and the third part of identically functionalized calix[4]- and calix[6]arenes, respectively.

The macrocyclic compounds 3, 4 and 7 described above present the inhibiting benzenesulfonamide units linked to the phenolic oxygens and then located at the opposite site with respect to the calixarene hydrophobic cavity. Therefore, with
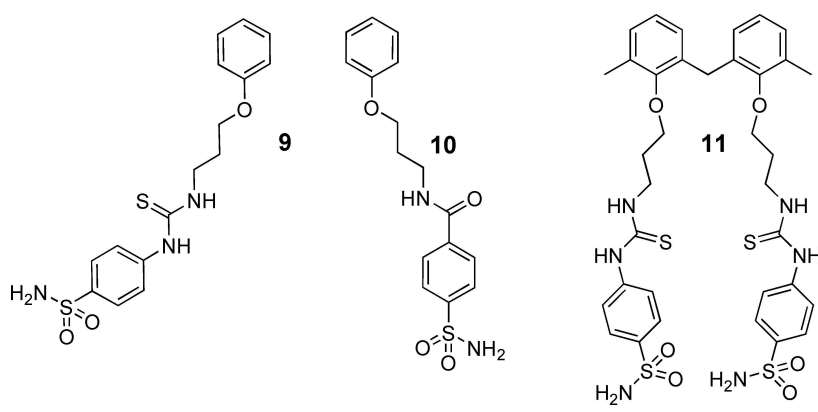

Figure 1. Mono- and divalent ligands. the aim of exploiting a possible synergy between the sulfonamide unit and the macrocyclic cavity in the interaction with the enzyme, we designed and prepared two derivatives, $16 \mathrm{a}$ and $16 \mathrm{~b}$ (Scheme 3 ), bearing a single sulfonamide moiety at the upper rim. In addition, in order to explore a possible role of the cavity in CA binding, while $16 \mathrm{~b}$ is blocked in cone geometry by the ethoxyethyl chains at the lower rim, 16a is conformationally mobile as a consequence of the reduced steric hindrance of the methoxy groups. In the latter compound $16 a$, the hydrophobic cavity is then not well fixed and preorganized as, on the contrary, in cone calixarenes.

For their synthesis, the two mononitro derivatives $12 \mathrm{a}, \mathrm{b}$ were reduced to the corresponding amino $13 a, b$, which were conjugated with the Boc protected $\gamma$-aminobutyric acid (GABA) to obtain $14 \mathrm{a}, \mathrm{b}$. GABA was selected as linker to maintain the distance between the calixarene scaffold and the sulfonamide unit comparable with that used in the previous derivatives. After deprotection of $14 a, b$ from Boc, the amines $15 a, b$ were connected to the benzensulfonamide unit through the generation of the thiourea spacer, giving $16 a, b$.

Carbonic anhydrase inhibition activity. The synthesized compounds were tested as inhibitors towards three different isoforms of human Carbonic Anhydrases (hCAs), hCA I, II and IX, determining the inhibition constant $\left(\mathrm{K}_{\mathrm{i}}\right)$ values through a stopped-flow technique. Acetazolamide (AAZ), a well-known topic drug for the treatment of glaucoma caused by CAs dysregulation, was used as reference compound. The enzyme inhibition activity was determined by assaying the CA catalyzed hydration of $\mathrm{CO}_{2}$ (Table 1). ${ }^{[23]}$

Since the calixarene derivatives functionalized with sulfonamide units resulted poorly soluble in water, we investigated their solubility in conditions compatible with the inhibition tests. We thus prepared samples dissolving the ligands in DMSO and adding water $\left(\mathrm{DMSO} / \mathrm{H}_{2} \mathrm{O} 1 / 9\right)$ to $10 \mathrm{mM}$ concentration and

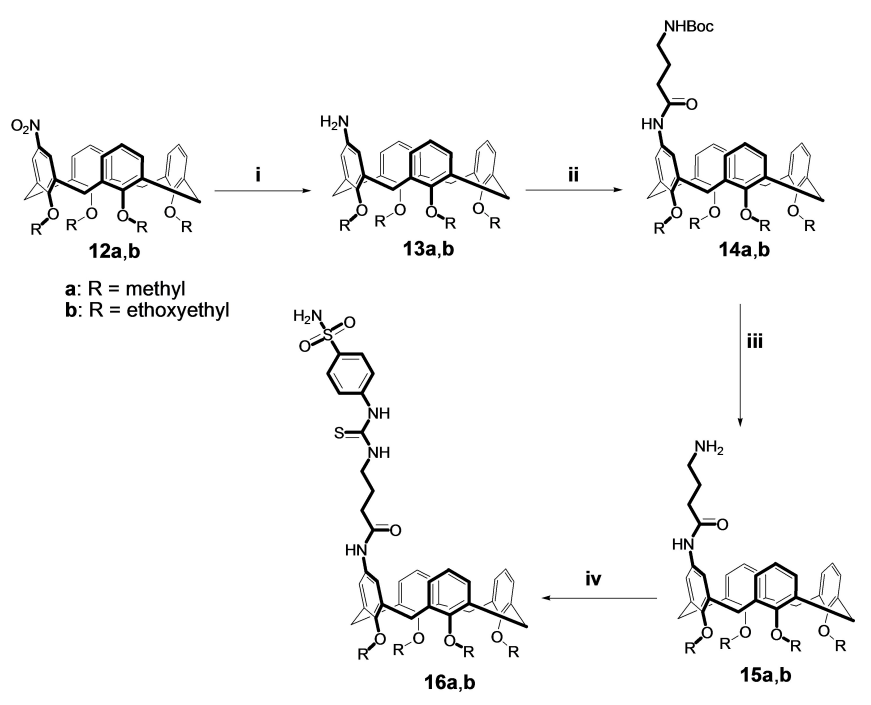

Scheme 3. Reagents and conditions: i) $\mathrm{NH}_{2} \mathrm{NH}_{2} \cdot \mathrm{H}_{2} \mathrm{O}, 10 \% \mathrm{Pd} / \mathrm{C}$ (catalytic amount), reflux; ii) EDC, Boc-GABA; iii) TES, TFA, dry DCM, rt, 4 h; vii) DIPEA, 4-isothiocyanatebenzensulfonamide. 
Table 1. Inhibition data of hCA I, hCA II, hCAIX with the synthesized compounds and the standard sulfonamide inhibitor acetazolamide (AAZ) by a stopped flow $\mathrm{CO}_{2}$ hydrase assay. ${ }^{[23]}$

\begin{tabular}{lllll} 
Compound & valency & $\begin{array}{l}\mathrm{K}_{\mathrm{i}}[\mu \mathrm{M}]^{[a]} \\
\text { hCA }\end{array}$ & hCA II & hCA IX \\
\hline $\mathbf{3 a}$ & 1 & $>100$ & $7.38 \pm 0.73$ & $1.98 \pm 0.16$ \\
$\mathbf{3 b}$ & 1 & $5.91 \pm 0.58$ & $3.09 \pm 0.14$ & $1.49 \pm 0.13$ \\
$\mathbf{4 b}$ & 1 & $5.57 \pm 0.42$ & $0.90 \pm 0.06$ & $1.77 \pm 0.09$ \\
$\mathbf{3 c}$ & 2 & $5.52 \pm 0.25$ & $0.53 \pm 0.03$ & $1.11 \pm 0.09$ \\
$\mathbf{4 c}$ & 2 & $8.29 \pm 0.56$ & $1.21 \pm 0.08$ & $0.25 \pm 0.01$ \\
$\mathbf{4 d}$ & 2 & $2.69 \pm 0.12$ & $0.34 \pm 0.03$ & $2.18 \pm 0.11$ \\
$\mathbf{3 e}$ & 4 & $8.57 \pm 0.65$ & $3.88 \pm 0.40$ & $4.03 \pm 0.36$ \\
$\mathbf{3 f}$ & 6 & $3.35 \pm 0.26$ & $0.50 \pm 0.03$ & $0.87 \pm 0.07$ \\
$\mathbf{4 f}$ & 6 & $5.95 \pm 0.62$ & $2.40 \pm 0.22$ & $0.82 \pm 0.088$ \\
$\mathbf{7}$ & 4 & $0.54 \pm 0.04$ & $0.40 \pm 0.03$ & $1.80 \pm 0.19$ \\
$\mathbf{1 6}$ & 1 & $3.75 \pm 0.37$ & $0.86 \pm 0.08$ & $1.66 \pm 0.14$ \\
$\mathbf{1 6} \mathbf{b}$ & 1 & $0.085 \pm 0.008$ & $0.048 \pm 0.005$ & $1.94 \pm 0.11$ \\
$\mathbf{9}$ & 1 & $0.008 \pm 0.0008$ & $0.008 \pm 0.0003$ & $0.029 \pm 0.002$ \\
$\mathbf{1 0}$ & 1 & $0.008 \pm 0.0007$ & $0.004 \pm 0.0002$ & $0.022 \pm 0.0009$ \\
$\mathbf{1 1}$ & 2 & $0.42 \pm 0.04$ & $0.089 \pm 0.006$ & $0.67 \pm 0.05$ \\
$\mathbf{A A Z}$ & 1 & $0.25 \pm 0.01$ & $0.012 \pm 0.0007$ & $0.025 \pm 0.001$ \\
\hline
\end{tabular}

[a] Mean from 3 different assays.

we diluted them with the assay buffer, never observing any precipitation.

The monovalent ligands 9 and 10 resulted the most potent inhibitors among the series, even more than $A A Z$, but they did not show any substantial selectivity among the three CAs considered in our study. In particular, for both derivatives, $\mathrm{K}_{\mathrm{i}}$ against hCA I interestingly resulted 30 -fold lower than that of the drug $\left(\mathrm{K}_{\mathrm{i}}=0.25 \mu \mathrm{M}\right)$. The behavior of the two compounds suggests that amide and thiourea units linking the benzenesulfonamide are substantially equivalent in the economy of the binding to the enzyme active site. These two compounds appear significantly more active than other sulfonamide containing inhibitors with similar structure. ${ }^{[24]}$

The $K_{i}$ values for the divalent derivative 11 are still in the $n M$ range, but they are 10-50 fold higher than the corresponding ones found for 9 and 10. Evidently, the two connected monomers not only do not work independently without any cooperativity and synergy, but are of mutual hindrance in the interaction with the active site. Multivalency is not working with this compound and the result substantially anticipates those found for the calixarene-based derivatives. Examining the collected values for the macrocycle series, no improvement in inhibition ability is in fact observed with respect to the monovalent ligands. Among the macrocyclic ligands, also no significant advantages are gained by increasing the number of sulfonamide units onto the calixarene scaffold. This is clear for example by comparing ligands $\mathbf{3 a}$ (monovalent), $3 \mathrm{c}$ (divalent), $\mathbf{3 e}$ (tetravalent) and $\mathbf{3} \mathbf{f}$ (hexavalent). Indeed, a significant reduction of the potency is evident for all the calixarene derivatives compared with 9 and 10. Nevertheless, in nearly all the cases the observed $\mathrm{K}_{\mathrm{i}}$ are at least in the $\mu \mathrm{M}$ range showing that these calixarenes, in general, are by far (2-3 order of magnitude) better CA inhibitors than those previously synthesized. ${ }^{[21]}$ Moreover, in not a few cases, their $K_{i}$ is in the $n M$ range highlighting a remarkable inhibition capability. In perspective, this is important considering the possibility of exploiting the macrocycle for the introduction of additional functions, such as covalently or non-covalently linked probes, responsive units, targeting species, pharmacophores, which would confer to the ligands additional tasks and properties. For instance, it is noteworthy the inhibition activity of $4 b\left(K_{i}=0.9 \mu M\right), 3 c\left(K_{i}=0.5 \mu M\right)$, $\mathbf{4 d}\left(\mathrm{K}_{\mathrm{i}}=0.3 \mu \mathrm{M}\right)$ and $\mathbf{3} \mathbf{f}\left(\mathrm{K}_{\mathrm{i}}=0.5 \mu \mathrm{M}\right)$ against $\mathrm{hCA}$ II, even if also for these macrocyclic compounds there is neither an evident influence of the valency or a regular advantage to have thiourea or amide spacer. Analogously, no systematic differences are distinguishable between calixarenes functionalized at the lower rim with propyl or ethoxyethyl chains.

One of the less active inhibitors is the tetravalent $3 \mathrm{e}$ probably as a consequence of the high level of crowding due to the cone geometry that would seem impair a favorable approach of the ligand to the enzyme and proper threading of the benzensulfonamide group into the enzyme funnel. Based on opposite considerations, not surprising are then the lower $K_{i}$ values found for the hexamer $\mathbf{3} \mathbf{f}$. Being conformationally mobile, this can in fact reduce the steric hindrance freely orienting the single arms in different directions and adapting its structure to the active site requests for a better interaction. Similarly, the 1,3-alternate tetravalent 7, although blocked in a rigid conformation, is more efficient than the tetravalent $3 \mathrm{e}$ (cone) being the sulfonamide units projected two by two towards opposite directions of the space. The reduced crowding of sulfonamide units seems thus to be responsible for the inhibition activity improvement of 7 respect to $3 \mathrm{e}$, rather than the possibility of putting into play the simultaneous binding to two or more CAs units. This multivalent interaction seems, in fact, not to occur, as later evidenced by the X-ray crystal structure analysis performed on the complex of $\mathbf{7}$ with hCA II (see below).

It is interesting the comparison among the monovalent ligands $4 b, 16 a$ and $16 b$, the first endowed with a cavity and an active unit diverging from it, the second being conformationally mobile and lacking a preorganized cavity, the third one with the cavity and the active unit adjacent one to the other. Particularly significant are the low $\mathrm{nM}$ range $\mathrm{K}_{\mathrm{i}}$ of compound $16 \mathrm{~b}$ against hCA I $\left(K_{i}=85 n M\right)$ and hCA II $\left(K_{i}=48 \mathrm{nM}\right)$. In the case of hCA I, $16 \mathrm{~b}$ is even more efficient than $A A Z\left(K_{i}=250 \mathrm{nM}\right)$ and it shows also a significant selectivity for hCA I and II over hCA IX $\left(\mathrm{K}_{\mathrm{i}}=\right.$ $1.9 \mu \mathrm{M}$ ) with a difference in $\mathrm{K}_{\mathrm{i}}$ of around two orders of magnitude. The presence of the ethoxyethyl chains at the lower rim of $\mathbf{4 b}$, in the same region where the benzensulfonamide unit is located, impairs an effective binding of the ligand to the active site, while in $16 \mathrm{~b}$ no other chains are linked to the upper rim of the calixarene. On the other hand, the conformationally mobile derivative $16 \mathrm{a}$, bearing the active sulfonamide unit at the upper rim as $16 \mathrm{~b}$, showed Ki values essentially equivalent to $4 \mathrm{~b}$ against all three CAs. This could suggest the involvement in the binding process of the cavity or at least a role of the scaffold preorganization characterizing $16 \mathrm{~b}$.

$X$-ray structures and molecular modeling studies. The structure of the complex between 9 and hCA II was solved by X-ray crystallography (Figure 2). Inspection of the initial Fo-Fc electron density maps immediately revealed the successful binding of the inhibitor in the active site, in which the ligand sulfonamide moiety showed the expected interaction to the zinc ion and residues nearby ${ }^{[25]}$ The deprotonated nitrogen atom displaces the hydroxide ion/water molecule present in the native enzyme and 


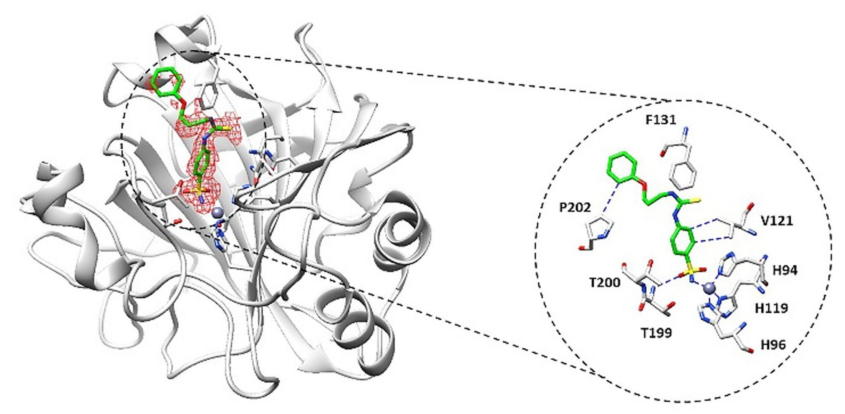

Figure 2. hCA II-9 complex (PDB: 7A6V). Compound 9 (in green) is showed as $\sigma A$-weighted $\mid$ Fo-Fc $\mid$ density map at $2.0 \sigma$.

coordinates the zinc ion with a tetrahedral geometry, while additional hydrogen bond interactions with residue Thr199 further contribute to stabilize the binding (Figure 2). The benzene ring bearing the sulfonamide occupies the hydrophobic region of the active site delimited by Phe131, Val121, Leu198, and Pro202, establishing hydrophobic interactions with these residues, in particular Val121, that stabilize further the complex. Moreover, Pro202 establishes a hydrophobic interaction with the phenolic ring of inhibitor 9 .

It should be stressed that single crystals were also grown for hCA II complexes with derivatives $4 \mathrm{~b}, 7$ and $\mathbf{1 6} \mathrm{b}$. However, for all of them, it was not possible to achieve the complete resolution of the ligand structure bound to the enzyme active site. Accurate atomic positions could be only detected for the benzensulfonamide moiety, the phenolic unit connected to it and the spacer in between, while the remaining structure of the macrocyclic scaffold and the other linked chains was not identifiable (e.g. Figure S53). Nonetheless, these experiments pointed out a 1:1 ligand:enzyme stoichiometry for the tetravalent derivative $\mathbf{7}$ and the clear evidence that the synthesized calixarene-based ligands can inhibit hCA II by a zinc coordination mechanism and not by generic, aspecific interactions.

Thus, in silico docking investigation on cone $(3 b, 16 b)$ and 1,3-alternate (7) calix[4]arenes were performed for completing the partial results achieved by experimental structural studies to describe more in detail the binding mode of the macrocyclic derivatives to hCA II. The three compounds were selected because considered representative of the different types of investigated macrocyclic ligands, having $\mathbf{3} \mathbf{b}$ the sulfonamide unit linked to the lower rim through an amide bond, 7 multiple copies of sulfonamide linked through the thiourea, and $16 \mathrm{~b}$ the sulfonamide at the upper rim. According to the experimental results, all docking solutions coherently located the ligand benzensulfonamide group inside the hCA II active sites. The sulfonamide binds to the zinc ion in its anionic form and completes the tetracoordination sphere of the metal ion. The coordination around the $\mathrm{Zn}^{2+}$ is strengthened by two $\mathrm{H}$-bonds formed by the sulfonamide $\mathrm{NH}$ and $\mathrm{S}=\mathrm{O}$ portions with the hydroxyl and the $\mathrm{NH}$ groups of T199 side chain and backbone, respectively. Dockings were integrated by MD simulations to validate and refine the ligand/ target adducts and to test the stability of the poses (Figures 3 and S54_MM1). For all investigated compounds the sulfonamide

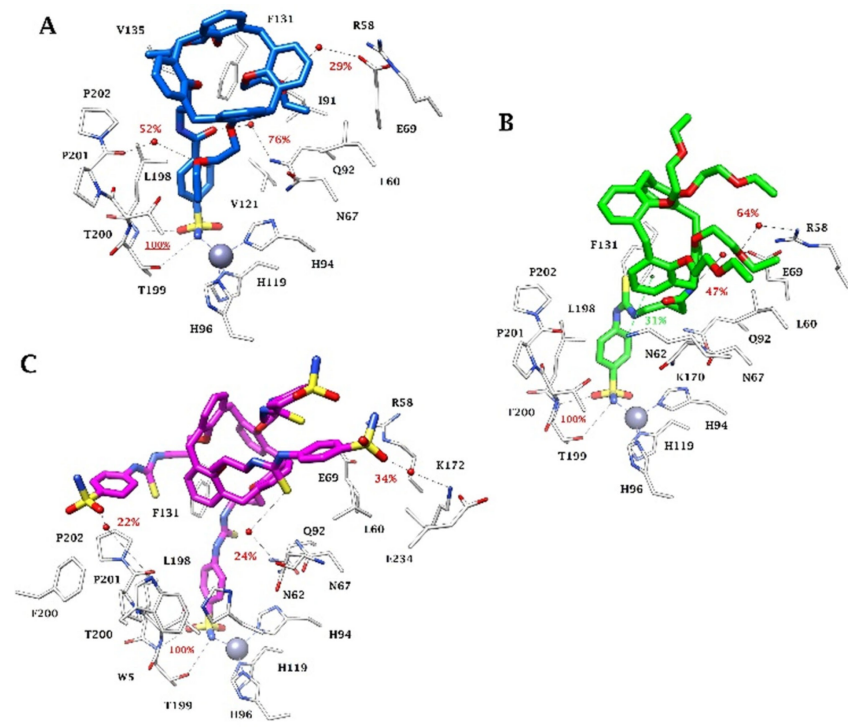

Figure 3. Main binding mode observed along the 100 ns MD simulations for calixarenes A) $\mathbf{3 b}$, B) $\mathbf{1 6} \mathrm{b}$ and C) $\mathbf{7}$ within the hCA II active site. $\mathrm{H}$-bond and $\pi$-cation interactions are depicted as black and green dashed lines, respectively. Water molecules are shown as red spheres. The persistence of interactions is reported as a percentage (red or green labels).

moiety maintains a stable coordination interaction with the zinc ion, which persists for the entire MD simulation (100 ns, percentage of persistence $100 \%)$.

In the most favorable bound pose of derivative $\mathbf{3} \mathbf{b}$ within the hCA II binding pocket, the cone calixarene moiety locates at the middle of the enzymatic cavity, preferentially moving towards the hydrophilic half of the active site during the MD simulations and forming contacts between the 2-ethoxyethyl pendants and both the hydrophilic and hydrophobic halves of the cleft (Figures $3 \mathrm{~A}$ and S54_MM1). In particular, the amide $\mathrm{CO}$ in between the benzensulfonamide moiety and the calixarene scaffold is firmly water-bridged with the Q92 side chain $(\mathrm{C}=\mathrm{O} \cdots \mathrm{HOH} \cdot \ldots \mathrm{HN}, 76 \%$ stable over the MD time). Also the 2-ethoxyethyl groups at the bottom (lower) rim of the cone are involved in water-bridged $\mathrm{H}$ bonds occurring between the ether oxygen atoms and the backbone $\mathrm{C}=\mathrm{O}$ of $\mathrm{P} 201(>\mathrm{O} \cdots \mathrm{HOH} \cdots \mathrm{O}=\mathrm{C}, 52 \%)$ and the E69 carboxylate ( $\left.>\mathrm{O} \cdots \mathrm{HOH}^{-. .-}{ }^{-} \mathrm{OOC}, 29 \%\right)$.

The analysis of the hCA II-4b complex seems to rule out an adverse impact of ethoxyethyl chains on the complexation. The sulfonamide unit, in fact, appears well located in the active site funnel interacting with zinc ion through $\mathrm{NH}^{-}$group and with Thr199 $\mathrm{NH}$ through $\mathrm{S}=\mathrm{O}$ group. One of the thioureido $\mathrm{NH}$ group is hydrogen bonded to a water molecule, in turn interacting with Thr200 OH.

A hydrogen bond mediated by a water molecule also occurs

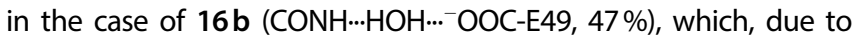
the greater length of the linker, folds back the calixarene scaffold towards the hydrophilic area of hCA II active site. Here the cone is stabilized by the interactions of different nature established with different residues, which, together, contribute to making the conformation stable for the entire MD simulation (Figures 3B and S54B_MM1). Three 2-ethoxyethyl tails per ligand protruded out- 
wards, whereas the other one was in water-bridge $\mathrm{H}$-bond distance with the side chain guanidine group of R58 (64\%).

The 1,3-alternate calix[4]arene 7 has one of the zinc-binder pendants involved in the metal coordination, mainly orienting the calixarene motif towards the hydrophilic half of the enzyme cavity. The other tioureidobenzosulfonamide pendants are involved in polar interactions with the target (Figures $3 \mathrm{C}$ and S54 C_MM1), establishing water-bridge $\mathrm{H}$-bonds with W5 (SO $\cdots \cdot \mathrm{HOH} \cdots \cdot \mathrm{HN}, 22 \%)$,

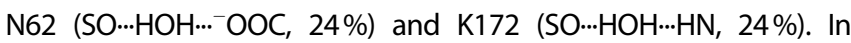
addition, two benzenesulfonamide moieties stack on each other and an intramolecular $\mathrm{H}$-bond is formed by one of them.

\section{Conclusions}

The different isoforms of CAs represent important therapeutic targets, due to the relationship between their overexpression or dysregulation and a wide variety of diseases. Searching for selective inhibitors of this class of enzymes and for scaffolds that, in perspective, could become the starting point for multifunctional systems able to combine CAs binding with additional theranostic activity, we have prepared calixarenes variably functionalized with benzensulfonamide, and phenol-based analogues.

The synthesized macrocycles including benzensulfonamide moieties were tested as inhibitors of hCA I, II and IX, and showed $\mathrm{K}_{\mathrm{i}}$ values in the micromolar to nanomolar range, accompanied in some cases by a noteworthy selectivity. For instance, calixarene 3 a showed a marked preference for hCA II and IX over hCA I, and $16 \mathrm{~b}$ for hCA I and II over hCA IX. It is relevant that the latter ligand showed comparable or even higher potency than the approved drug AAZ against hCA II and hCA I, respectively.

Although the X-ray crystal structure of the complex between any macrocyclic ligand and hCA II could not be completely solved, for three of them, $\mathbf{4 b}, \mathbf{7}$ and $\mathbf{1 6 b}$, it was possible to establish the unequivocal position of the chain terminating with the sulfonamide unit located in the hCA II active site. Molecular modeling studies confirmed the mode of binding and, together with the other data from inhibition assays and X-ray crystallography, allow to conclude that calixarenes represent a suitable platform to build efficient CAs ligands able of recruiting the enzyme active site and with potential selectivity among the different isoforms. Moreover, the intrinsic nature of these macrocycles paves the way towards multifunctional derivatives able to combine, thanks to the introduction of further proper active units, the enzyme inhibition/ targeting with other medically relevant activities. Although for the derivatives here proposed multivalency does not seem to play a role, probably in part due to the shortness of the spacers introduced between the macrocyclic scaffolds and the benzensulfonamide groups, it cannot be ruled out that it can arise by using longer linkers. This aspect will also be the object of further investigation by our side.

Interestingly, both the monovalent models 9 and 10, despite their rather simple structure, resulted very potent inhibitors of the three studied CAs, showing in particular for CA I much higher efficiency than AAZ. Also, these two molecules can thus be the starting point for further and more sophisticated developments.

\section{Experimental Section}

General information: All moisture-sensitive reactions were carried out under a nitrogen atmosphere. All dry solvents were prepared according to standard procedures and stored over 3 or $4 \AA$ molecular sieves. All the other commercially available reagents were used without further purification. TLC were performed using commercially available plates of silica gel (Merck $60 \mathrm{~F}_{254}$ on aluminium support) and revealed using UV light or staining reagents: $\mathrm{FeCl}_{3}\left(1 \%\right.$ in $\left.\mathrm{H}_{2} \mathrm{O} / \mathrm{CH}_{3} \mathrm{OH} 1 / 1\right)$, ninhydrin $(5 \%$ in EtOH), basic solution of $\mathrm{KMnO}_{4}\left(0.75 \%\right.$ in $\left.\mathrm{H}_{2} \mathrm{O}\right)$. Flash chromatography was performed on 32-63 $\mu \mathrm{m}, 60 \AA$ Merck silica gel. ' $\mathrm{H}$ NMR (300 or $400 \mathrm{MHz}$ ) and ${ }^{13} \mathrm{C}$ NMR spectra (75 or $100 \mathrm{MHz}$ ) were recorded on Bruker AV300 and AV400 spectrometers using partially deuterated solvents as internal standards. All ${ }^{13} \mathrm{C}$ NMR were performed with proton decoupling. Mass spectra were recorded in Electrospray lonization (ESI) mode using a LTQ Orbitrap XL spectrometer. Microwave assisted reactions were performed in a CEM Discover SP system.

25,26,27-Tris(2-ethoxyethoxy)calix[4]arene, ${ }^{[26]}$ 25,27-bis(2-ethoxyethoxy)calix[4]arene, ${ }^{[27]} \quad 25-(3-\mathrm{N}$-phthalimido)propoxy-26,27,28tripropoxycalix[4]arene ${ }^{[28]} \quad(1 \mathrm{a}), \quad 25,26,27,28$-tetrakis-(3-Nphthalimido)propoxycalix[4]arene ${ }^{[29]}$ (1 e), 25,27-dipropoxy-26,28bis-(3-N-phthalimido)propoxycalix[4]arene ${ }^{[28]}$

$37,38,39,40,41,42$-hexakis(3-N-phtalimido)propoxycalix[6]arene ${ }^{[30]}$

(1 f), 25-(3-amino)propoxy-26,27,28-tripropoxycalix[4]arene ${ }^{[28]}$ (2 a), 25,26,27,28-tetrakis(3-amino)propoxycalix[4]arene ${ }^{[29]}$ (2 e), 25,27-dipropoxy-26,28-bis(3-aminopropoxy)calix[4]arene ${ }^{[28]} \quad$ (2c), $37,38,39,40,41,42$-hexakis(3-aminopropoxy)calix[6]arene $e^{[30]} \quad(2 \mathrm{f}), 5-$ nitro-25,26,27,28-tetrakis(2-ethoxyethoxy)calix[4]arene ${ }^{[26]}$ (12 b), 5amino-25,26,27,28-tetrakis(2-ethoxyethoxy)calix[4]arene ${ }^{[31]}(13 \mathrm{~b}), 1$ phenoxy-3-aminopropane ${ }^{[32]}(8)$, bis[(2-(3'-amino)propoxy-3-meth$\mathrm{yl})$ phenyl]methane ${ }^{[19]}$ are already known and their characterization already published. They were prepared accordingly with the literature procedures. The details of the synthetic procedures and characterization of the new compounds are reported in Supporting Information.

General procedure for the coupling between the amino derivatives and 4-sulfamoylbenzoic acid: In a 2-necked round-bottom flask, 4-sulfamoylbenzoic acid (2.0 equiv. $\times$ each amine unit) and DIPEA (2.4 equiv. $\times$ reactive unit) were stirred in dry $\mathrm{CH}_{2} \mathrm{Cl}_{2}$ for 10 minutes at room temperature. Then EDC was added and the resulting mixture was stirred for 10 minutes. This mixture was hence dropped in a solution in dry $\mathrm{CH}_{2} \mathrm{Cl}_{2}$ or dry DMF of the amino derivative and the reaction was stirred for $4-18 \mathrm{~h}$ at $\mathrm{rt}$ if not otherwise specified. To obtain compounds $3 \mathrm{~b}$ and $3 \mathrm{c}$, the reaction was performed in a microwave reactor $\left(2\right.$ cycles, $\mathrm{T}=80^{\circ} \mathrm{C}$, ramp time $=3$ minutes, hold time $=2 \mathrm{~h}, \mathrm{P}=200 \mathrm{psi}$, potency $=200 \mathrm{~W}$ ).

General procedure for the coupling between the amino derivatives and 4-isothiocyanatebenzenesulfonamide: In a 2-necked round-bottom flask, 4-isothiocyanatobenzenesulfonamide (2.0 equiv. $\times$ amine unit) and the amino derivative are reacted in dry $\mathrm{CH}_{2} \mathrm{Cl}_{2}$ or dry DMF or in a mixture of both for $4 \mathrm{~h}-4$ days at it in presence of DIPEA (2.0 equiv. $\times$ reactive unit).

In vitro carbonic anhydrase inhibition assay: The $\mathrm{CA}$-catalyzed $\mathrm{CO}_{2}$ hydration activity was performed on an Applied Photophysics stopped-flow instrument using phenol red (at a concentration of $0.2 \mathrm{mM}$ ) as a pH indicator with $20 \mathrm{mM}$ Hepes ( $\mathrm{pH} 7.5)$ as the buffer, $20 \mathrm{mM} \mathrm{Na}_{2} \mathrm{SO}_{4}$, and following the initial rates of the CA-catalyzed $\mathrm{CO}_{2}$ hydration reaction for a period of 10-100 $\mathrm{s}$ and working at the maximum absorbance of $557 \mathrm{~nm} \cdot{ }^{[23]}$ The $\mathrm{CO}_{2}$ concentrations ranged from 1.7 to $17 \mathrm{mM}$. For each inhibitor six traces of the initial 5-10\% of the reaction have been used in order to determine the initial velocity. The uncatalyzed reaction rates were determined in the 
same manner and subtracted from the total observed rates. Stock solutions of the inhibitors $(10 \mathrm{mM})$ were prepared dissolving the compounds in DMSO and adding distilled-deionized water (1:9) to a $10 \mathrm{mM}$ concentration. Dilutions up to $0.01 \mathrm{nM}$ were done thereafter with the assay buffer. Solutions containing inhibitor and enzyme were preincubated for $15 \mathrm{~min}$ at room temperature prior to assay in order to allow the formation of the E-I complex. The inhibition constants were obtained as nonlinear least-squares protocols using PRISM 3 and are the mean from at least three different measurements. All hCAs were recombinant ones and were obtained in house. ${ }^{[33]}$

Protein X-ray crystallography: Crystals were obtained using the hanging drop vapor diffusion method using 24 well Linbro plate. $2 \mu \mathrm{l}$ of $10 \mathrm{mg} / \mathrm{ml}$ solution of hCA II in Tris- $\mathrm{HCl} \mathrm{pH}=8.0$ were mixed with of a solution of $1.5,1.6$ and $1.7 \mathrm{M}$ sodium citrate, $50 \mathrm{mM}$ Tris $\mathrm{pH} 8.0$ and were equilibrated against $500 \mu \mathrm{l}$ of the same solution at 296 K. Crystals of the protein grew in few days. Afterwards hCA II crystals were soaked in $10 \mathrm{mM}$ inhibitor solution for 3 days. The crystal was flash-frozen at $100 \mathrm{~K}$ using a solution obtained by adding $15 \%(\mathrm{v} / \mathrm{v})$ glycerol to the mother liquor solution as cryoprotectant. Data on crystals of the complexes were collected using a D8 Venture instrument (Bruker) equipped with a PHOTON III detector at CRIST (Florence, Italy) using the CuKa wavelength of $1.54184 \AA$ A. Data were integrated and scaled using the program XDS. ${ }^{[34]}$

X-ray crystal structure determination: The crystal structure of hCA II (PDB accession code: 4FIK) without solvent molecules and other heteroatoms was used to obtain initial phases of the structures using Refmac5. ${ }^{[35]} 5 \%$ of the unique reflections were selected randomly and excluded from the refinement data set for the purpose of Rfree calculations. The initial $|\mathrm{Fo}-\mathrm{Fc}|$ difference electron density maps unambiguously showed the inhibitor molecules. Atomic models for inhibitors were calculated and energy minimized using the program JLigand $1.0 .40 .^{[36]}$ Refinements proceeded using normal protocols of positional, isotropic atomic displacement parameters alternating with manual building of the models using COOT. ${ }^{[37]}$ Solvent molecules were introduced automatically using the program ARP. ${ }^{[38]}$ The quality of the final models were assessed with COOT and RAMPAGE. ${ }^{[39]}$ Atomic coordinates were deposited in the Protein Data Bank, Deposition Number: $7 \mathrm{A6V}$; Supporting Information contains the supplementary crystallographic data for this paper. These data are provided free of charge by the Protein Data Bank (https://www.rcsb.org/structure), Graphical representations were generated with Chimera. ${ }^{[40]}$

Molecular modeling: The crystal structure of hCA II (PDB 3K34) ${ }^{[41]}$ was prepared using the Protein Preparation Wizard tool implemented in the Schrödinger suite. ${ }^{[42]}$ The energy minimization protocol with a root mean square deviation (RMSD) value of $0.30 \AA$ was applied using force field OPLS3e.

Ligands were built starting from the crystal structure WUVKON and XUZLOU retrived from CSD. ${ }^{[43]}$ The coordinates were properly modified to obtain $\mathbf{4 b}, 7$ and $16 \mathrm{~b}$. The compounds were submitted to QM geometry optimization (B3LYP/6-31G* + ) and ESP charges calculation with Jaguar. ${ }^{[42 h]}$ The software Glide was used for docking $^{[42 f]}$ using ligand QM charges. Grids were centered on the centroids of the zinc-coordinating residues and ligands were docked using standard precision mode (SP). The best pose of compounds $\mathbf{7 b}, \mathbf{1 1}$ and $\mathbf{1 5} \mathrm{b}$ within hCA II active site, evaluated in terms of anchorage, hydrogen bond interactions and hydrophobic contacts, was submitted to MD simulation using Desmond and the OPL3e force field ${ }^{[42 g, 44]}$ performed in triplicate. Specifically, the system was solvated in an orthorhombic box using TIP4PEW water molecules, extended $15 \AA$ away from any protein atom, using the ligand $\mathrm{QM}$ charges. It was neutralized by adding chlorine and sodium ions. The simulation protocol included a starting relaxation step followed by a final production phase of $100 \mathrm{~ns}$. In particular, the relaxation step comprised the following: (a) a stage of 100 ps at $10 \mathrm{~K}$ retaining the harmonic restraints on the solute heavy atoms (force constant of $50.0 \mathrm{kcal} \mathrm{mol}^{-1} \AA^{-2}$ ) using the NPT ensemble with Brownian dynamics; (b) a stage of $12 \mathrm{ps}$ at $10 \mathrm{~K}$ with harmonic restraints on the solute heavy atoms (force constant of $50.0 \mathrm{kcalmol}^{-1} \AA^{-2}$ ), using the NVT ensemble and Berendsen thermostat; (c) a stage of $12 \mathrm{ps}$ at $10 \mathrm{~K}$ and $1 \mathrm{~atm}$, retaining the harmonic restraints and using the NPT ensemble and Berendsen thermostat and barostat; ( $f$ a stage of $12 \mathrm{ps}$ at $300 \mathrm{~K}$ and $1 \mathrm{~atm}$, retaining the harmonic restraints and using the NPT ensemble and Berendsen thermostat and barostat; (g) a final 24 ps stage at $300 \mathrm{~K}$ and $1 \mathrm{~atm}$ without harmonic restraints, using the NPT Berendsen thermostat and barostat. The final production phase of MD was run using a canonical NPT Berendsen ensemble at temperature $300 \mathrm{~K}$. During the MD simulation, a time step of $2 \mathrm{fs}$ was used while constraining the bond lengths of hydrogen atoms with the $M$ SHAKE algorithm. The atomic coordinates of the system were saved every 100 ps along the MD trajectory. Protein and ligand RMSD values, ligand torsions evolution and occupancy of intermolecular hydrogen bonds and hydrophobic contacts were computed along with the production phase of the MD simulation with the Simulation Interaction Diagram tools implemented in Maestro and discussed as a mean of the three MD simulation replicas.

\section{Acknowledgements}

F. S. and A. C. wish to acknowledge the Centro Interdipartimentale Misure (CIM) for the use of NMR and Mass Spectrometry facilities. The research from Parma Laboratory was supported by the Italian Ministry of University and Research projects (COMPHUB initiative, Departments of Excellence Program and BacHound PRIN 2017E44A9P). Research from the Florence laboratory was financed by the Italian Ministry for University and Research (MUR), grants PRIN prot. 2017XYBP2R and FISR2019_ 04819 BacCAD to C. T. S.

\section{Conflict of Interest}

The authors declare no conflict of interest.

Keywords: benzensulfonamide calixarenes carbonic anhydrases - carbonic anhydrases X-ray crystal structure enzyme inhibition

[1] T. H. Maren, Physiol. Rev. 1967, 47, 595-781.

[2] C. T. Supuran, Biochem. J. 2016, 473, 2023-2032.

[3] C. T. Supuran, J. Enzyme Inhib. Med. Chem. 2012, 27, 759-772.

[4] a) C. T. Supuran, Nature Rev. Drug Dis. 2008, 7, 168-181; b) C. T. Supuran Clin. Sci. 2021, 135, 1233-1249.

[5] E. Berrino, L. Milazzo, L. Micheli, D. Vullo, A. Angeli, M. Bozdag, A. Nocentini, M. Menicatti, G. Bartolucci, L. di Cesare Mannelli, C. Ghelardini, C. T. Supuran, F. Carta, J. Med. Chem. 2019, 62, 7233-7249.

[6] M. Gallorini, A. C. Berardi, A. Ricci, C. Antonetti Lamorgese Passeri, S. Zara, F. Oliva, A. Cataldi, F. Carta, S. Carradori, Biomedicine 2021, 9, 141157.

[7] a) N. Krall, F. Pretto, D. Neri, Chem. Sci. 2014, 5, 3640-3644; b) N. Krall, F. Pretto, W. Decurtins, G. J. L. Bernardes, C. T. Supuran, D. Neri, Angew. 
Chem. Int. Ed. 2014, 53, 4231-4235; Angew. Chem. 2014, 126, 43154320.

[8] H. S. Jung, J. Han, H. Shi, S. Koo, H. Singh, H.-J. Kim, J. L. Sessler, J. Y. Lee, J.-H. Kim, J. S. Kim, J. Am. Chem. Soc. 2017, 139, 7595-7602.

[9] A. Angeli, L. Di Cesare Mannelli, C. Ghelardini, T. S. Peat, G. Bartolucci, M. Menicatti, F. Carta, C. T. Supuran, Eur. J. Med. Chem. 2019, 177, 188-197.

[10] a) J. A. Aaron, J. M. Chambers, K. M. Jude, L. Di Costanzo, I. J. Dmochowski, D. W. Christianson, J. Am. Chem. Soc. 2008, 130, 6942-6943; b) J. M. Chambers, P. Aru Hill, J. A. Aaron, Z. Han, D. W. Christianson, N. N. Kuzma, I. J. Dmochowski, J. Am. Chem. Soc. 2009, 131, 563-569.

[11] V. Alterio, A. Di Fiore, K. D'Ambrosio, C. T. Supuran, G. De Simone, Chem. Rev. 2012, 112, 4421-4468.

[12] a) M. Mammen, S.-K. Choi, G. M. Whitesides, Angew. Chem. Int. Ed. 1998 , 37, 2754-2794Angew. Chem. 1998, 110, 2908-2953; Angew. Chem. 1998, 110, 2908-2953; Angew. Chem. Int. Ed. 1998, 37, 2754-2794; b) C. Fasting, C. A. Schalley, M. Weber, O. Seitz, S. Hecht, B. Koksch, J. Dernedde, C. Graf, E.-W. Knapp, R. Haag, Angew. Chem. Int. Ed. Engl. 2012, 51, 10472-10498; Angew. Chem. 2012, 124, 10622-10650; Angew. Chem. Int. Ed. 2012, 51, 10472-10498; c) J. Huskens, L. J. Prins, R. Haag, B. J. Ravoo, in Multivalency: Concepts, Research \& Applications, Wiley, 2018.

[13] a) N. Kanfar, E. Bartolami, R. Zelli, A. Marra, J.-Y. Winum, S. Ulrich, P. Dumy, Org. Biomol. Chem. 2015, 13, 9894-9906; b) S. G. Gouin, Chem Eur. J. 2014, 20, 11616-11628; c) P. Compain, Chem. Rec. 2020, 20, 1022; d) A. Joosten, C. Decroocq, J. de Sousa, J. P. Schneider, E. Etamé, A. Bodlenner, T. D. Butters, P. Compain, ChemBioChem 2014, 15, 309-319; e) E. Howard, A. Cousido-Siah, M. L. Lepage, J.P. Schneider, A. Bodlenner, A. Mitschler, A. Meli, I. Izzo, H. A. Alvarez, A. Podjarny, P. Compain, Angew. Chem. Int. Ed. 2018, 57, 8002-8006; Angew. Chem. 2018, 130, 8134-8138.

[14] F. Carta, P. Dumy, C. T. Supuran, J.-Y. Winum, Int. J. Mol. Sci. 2019, 20, 5352-5365.

[15] M. Stiti, A. Cecchi, M. Rami, M. Abdaoui, V. Barragan-Montero, A. Scozzafava, Y. Guari, J.-Y. Winum, C. T. Supuran, J. Am. Chem. Soc. 2008 130, 16130-16131.

[16] M. Abellán-Flos, M. Tanç, C. T. Supuran, S. P. Vincent, Org. Biomol. Chem. 2015, 13, 7445-7451.

[17] a) S. M. Sebti, A. D. Hamilton, Oncogene 2000, 19, 6566-6573; b) V. Martos, S. C. Bell, E. Santos, E. Y. Isacoff, D. Trauner, J. de Mendoza, Proc Natl. Acad. Sci. USA 2009, 106, 10482-10486.

[18] a) S. Cecioni, R. Lalor, B. Blanchard, J. P. Praly, A. Imberty, S. E. Matthews, S. Vidal, Chem. Eur. J. 2009, 15, 13232-13240; b) S. André, F. Sansone, H. Kaltner, A. Casnati, J. Kopitz, H. J. Gabius, R. Ungaro, ChemBioChem 2008, 9, 1649-1661; c) J. Garcia-Hartjes, S. Bernardi, C. A. G. M. Weijers, T. Wennekes, M. Gilbert, F. Sansone, A. Casnati, H. Zuilhof, Org. Biomol. Chem. 2013, 11, 4340-4349.

[19] a) V. Bagnacani, F. Sansone, G. Donofrio, L. Baldini, A. Casnati, R. Ungaro, Org. Lett. 2008, 10, 3953-3956; b) V. Bagnacani, V. Franceschi, M. Bassi, M. Lomazzi, G. Donofrio, F. Sansone, A. Casnati, R. Ungaro, Nat Commun. 2013, 4:1721.

[20] a) M. Gómez-García, J. M. Benito, A.P. Butera, C. Ortiz Mellet, J. M. García Fernández, J. L. Jiménez Blanco, J. Org. Chem. 2012, 77, 12731288; b) M. González-Cuesta, D. Goyard, E. Nanba, K. Higaki, J. M. García Fernández, O. Renaudet, C. Ortiz Mellet, Chem. Commun. 2019, 55, 12845-12848; c) X. Ferhati, C. Matassini, M. G. Fabbrini, A. Goti, A. Morrone, F. Cardona, A. J. Moreno-Vargas, P. Paoli, Bioorg. Chem. 2019, $87,534-549$.

[21] M. Oguza, E. Kalay, S. Akocak, A. Nocentini, N. Lolak, M. Boga, M. Yilmaz, C. T. Supuran, J. Enzyme Inhib. Med. Chem. 2020, 35, 1215-1223.

[22] a) A. Scozzafava, L. Menabuoni, F. Mincione, F. Briganti, G. Mincione, C. T. Supuran, J. Med. Chem. 1999, 42, 2641-2650; b) K. D'Ambrosio, F.-Z. Smaine, F. Carta, G. De Simone, J.-Y. Winum, C. T. Supuran, J. Med. Chem. 2012, 55, 6776-6783; c) C. T. Supuran, Expert Opin. Drug Discovery 2020, 15, 671-686.

[23] R. G. Khalifah, J. Biol. Chem. 1971, 246, 2561-2573.

[24] A. M. Zhanga, N. Weia, X. F. Liua, M. G. Wub, G. S. Xuan, Russ. J. Bioorg. Chem. 2021, 47, 261-269.
[25] A. Angeli, D. Tanini, A. Capperucci, G. Malevolti, F. Turco, M. Ferraroni, C. T. Supuran, Bioorg. Chem. 2018, 81, 642-648.

[26] A. R. N.S. Royappa, M. Ayer, A. Fracassi, M.-O Ebert, S. Aroua, Y. Yamakoshi, Helv. Chim. Acta 2017, 100, e1600391.

[27] A. Ikeda, T. Tsudera, S. Shinkai, J. Org. Chem. 1997, 62, 3568-3574.

[28] L. Baldini, M. Melegari, V. Bagnacani, A. Casnati, E. Dalcanale, F. Sansone, R. Ungaro, J. Org. Chem. 2011, 76, 3720-3732.

[29] V. Bagnacani, V. Franceschi, L. Fantuzzi, F. Sansone, G. Donofrio, A. Casnati, R. Ungaro, Bioconjugate Chem. 2012, 23, 993-1002.

[30] A. Casnati, N. Della Ca', M. Fontanella, F. Sansone, F. Ugozzoli, R. Ungaro, K. Liger, J.-F. Dozol, Eur. J. Org. Chem. 2005, 2338-2348.

[31] J. D. Van Loom, R. G. Janssen, W. Verboom, D. N. Reinhoudt, Tetrahedron Lett. 1992, 33, 5125-5128.

[32] N. E. Behnke, R. Kielawa, D.-H. Kwon, D. H. Ess, L. Kürti, Org. Lett. 2018, 20, 8064-8068.

[33] a) A. Angeli, L. Di Cesare Mannelli, E. Lucarini, T. S. Peat, C. Ghelardini, C. T. Supuran, Eur. J. Med. Chem. 2018, 154, 210-219; b) O. Akgul, L. Di Cesare Mannelli, D. Vullo, A. Angeli, C. Ghelardini, G. Bartolucci, A. S. Alfawaz Altamimi, A. Scozzafava, C. T. Supuran, F. Carta, J. Med. Chem. 2018, 61, 4961-4977; c) L. Vats, V. Sharma, A. Angeli, R. Kumar, C. T. Supuran, P. K. Sharma, Eur. J. Med. Chem. 2018, 150, 678-686; d) E. Bruno, M. R. Buemi, A. Di Fiore, L. De Luca, S. Ferro, A. Angeli, R. Cirilli, D. Sadutto, V. Alterio, S. M. Monti, C. T. Supuran, G. De Simone, R. Gitto, J. Med. Chem. 2017, 60, 4316-4326.

[34] W. Kabsch, Acta Crystallogr. Sect. D 2010, 66, 125-132.

[35] G. N. Murshudov, P. Skubak, A. A. Lebedev, N. S. Pannu, R. A. Steiner, R. A. Nicholls, M. D. Winn, F. Long, A. A. Vagin, Acta Crystallogr. Sect. D 2011, 67, 355-367.

[36] A. A. Lebedev, P. Young, M. N. Isupov, O. V. Moroz, A. A. Vagin, G. N. Murshudov, Acta Crystallogr. Sect. D 2012, 68, 431-440.

[37] P. Emsley, B. Lohkamp, W. G. Scott, K. Cowtan, Acta Crystallogr. Sect. D 2010, 66, 486-501.

[38] V. S. Lamzin, A. Perrakis, K. S. Wilson, in Crystallography of Biological Macromolecules (Eds.: M. G. Rossmann, E. Arnold), Kluwer Academic Publishers, Dordrecht, The Netherlands, 2001, p. 720.

[39] S. C. Lovell, I. W. Davis, W. B. Arendall III, P. I. W. de Bakker, J. M. Word, M. G. Prisant, J. S. Richardson, D. C. Richardson, Proteins 2003, 50, 437450.

[40] E. F. Pettersen, T. D. Goddard, C. C. Huang, G. S. Couch, D. M. Greenblatt, E. C. Meng, T. E. Ferrin, J. Comput. Chem. 2004, 25, 1605-1612.

[41] a) C. A. Behnke, I. Le Trong, J. W. Godden, E. A. Merritt, D. C. Teller, J. Bajorath, R. E. Stenkampa, Acta Crystallogr. Sect. D 2010, 66, 616-627; b) RCSB Protein Data Bank: powerful new tools for exploring 3D structures of biological macromolecules for basic and applied research and education in fundamental biology, biomedicine, biotechnology, bioengineering and energy sciences. Nucleic Acids Res. 2021, 49, D437D451.

[42] Schrödinger Suite Release 2019-1, Schrödinger, LLC, New York, NY, 2019: a) Prime, v.5.5; b) Maestro v.11.9; c) Epik, v.4.7; d) Impact, v.8.2; e) Macromodel v.12.3; f) Glide, v.8.2; g) Desmond, v.5.7; h) Jaguar, v. 10.3 .

[43] F. H. Allen, Acta Crystallogr. Sect. B 2002, 58, 380-388. The CSD version used in this work was updated in 2021.

[44] a) A. Pustenko, A. Nocentini, P. Gratteri, A. Bonardi, I. Vozny, R. Žalubovskis, C. T. Supuran, J. Enzyme Inhib. Med. Chem. 2020, 35, 10111020; b) A. Bonardi, A. Nocentini, R. Cadoni, S. del Prete, P. Dumy, C Capasso, P. Gratteri, C. T. Supuran, J.-Y. Winum, ACS Med. Chem. Lett. 2020, 11, 2277-2284; C) A. Nocentini, A. Bonardi, P. Gratteri, B. Cerra, A. Gioiello, C. T. Supuran, J. Enzyme Inhib. Med. Chem. 2018, 33, 14531459.

Manuscript received: September 28, 2021

Accepted manuscript online: December 9, 2021

Version of record online: December 30, 2021 